\title{
Efficient, stable and high color rendering index white polymer light-emitting diodes by restraining the electron trapping
}

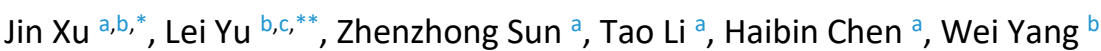 \\ ${ }^{a}$ Neutron Scattering Technical Engineering Research Center, School of Mechanical Engineering, Dongguan University of Technology, Dongguan, 523808, China ${ }^{b}$ \\ Institute of Polymer Optoelectronic Materials and Devices, State Key Laboratory of Luminescent Materials and Devices, South China University of Technology, \\ Guangzhou, 510640, China ${ }^{\mathrm{C}}$ Guangdong Juhua Printed Display Technology Co., Ltd, Guangzhou, 510700, China
}

\section{ARTICLEINFO ABSTRACT}

\section{Keywords:}

WPLED

Fluorescence

Polymer blends

Electron trapping

\begin{abstract}
Superior solid-state light source should possess not only a high device efficiency but also high color rendering index (CRI) and excellent color stability. Here, we obtained efficient, stable and high CRI white polymer light- emitting diodes (WPLEDs) through applying the well-chosen trichromatic polymers and restraining the electron trapping. The trichromatic polymers all possess the similar mainchain units, their EL spectra shows an equidistant distribution. Detailed studies revealed that the part of green and red emission in the WPLED derive from the electron trapping which led to the current-dependent spectra. Applied proper electron transporting layer can enhance the direct electron injection which resulted in the electron trapping being restrained and improving the color stability. The optimized WPLED got a $L E_{\max }$ of $10.17 \mathrm{~cd} \mathrm{~A}^{1}$ and $C R I$ of 92 , the CIE coordinates $(0.346,0.345)$ almost keep the same with the operating current density increasing from $10.53 \mathrm{~mA} \mathrm{~cm}{ }^{2}$ to $263.16 \mathrm{~mA} \mathrm{~cm}{ }^{2}$.
\end{abstract}

\section{Introduction}

Apart from the application of color display, the potential application of organic light emitting diodes (OLEDs) in the solid-state lightings have also attracted increasing interest due to its superior property of high efficiency, flexible and area light source [1-4]. Compared with the vapor-deposited white OLEDs, solution processed white polymer light-emitting diodes (WPLEDs) possess some obvious advantages in low-cost manufacturing process, large area fabrication, and better controllable doping concentrations [5-7]. It should be noted that as an excellent illumination light sources for industrial application, it should possess not only a high efficiency but also high color rendering index (CRI) and superior color stability. The CRI presents the light sources' ability of restoring the original color of object. Unfortunately, most of OLED light source reported have a CRI of below 90 [8-10].

Various approaches, such as single white light polymers [11,12], exciplex [13], multiple blending [14] and utilization of tandem device [15,16], have been reported to realize white emission. Thereinto, multiple blending should be the advisable means to achieve high CRI white emission considering the simple device structure especially for the solution processed. Normally, the mechanism of acquisition of white emission mainly includes the energy transfer and charge trapping for the multiple blending systems. For the former, the PL spectra of the host materials (blue) need to overlap with the absorption spectra of the guest (green/red) [17]. It's generally considered that the larger the overlap, the stronger the energy transfer. The content of guest materials' weights usually below $1 \%$ for the polymer blend system with stronger energy transfer, which make the EL spectra pretty sensitive to its concentration so that it is quite difficult to optimize for obtaining white emission with high CRI (>90) [18-20]. Unlike the energy transfer, the latter scenario requires the highest occupied molecular orbital (HOMO) or the lowest unoccupied molecular orbital (LUMO) levels of guest materials (green and red) lying in the band gap of host material (blue). But since the emission intensity ratio of host and guest tend to change along with the applied bias, it's pretty difficult to obtain stable spectrum based on the charge trapping [21,22]. Much efforts including low dopant concentration, inserting an insulating material and binary host have been done to overcome the issue of color stable stemming from the charge trapping [23-26], but in the meanwhile these measures

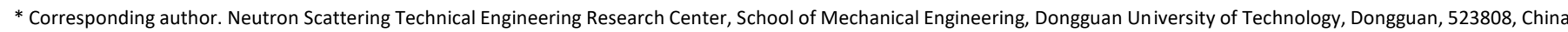

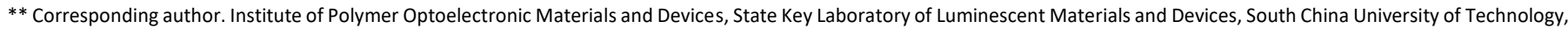
Guangzhou, 510640, China.E-mail address: xujin@dgut.edu.cn (J. Xu). https://doi.org/10.1016/j.orgel.2020.105785
}

Received 2 March 2020; Received in revised form 9 April 2020; Accepted 13 April 2020

Available online 20 April 2020

1566-1199/@ 2020 Elsevier B.V. All rights reserved. 
would inevitably affect the device efficiency or CRI value. Therefore, that obtaining white light source possess high efficiency, high CRI and stable spectrum at the same time seems to be a tricky work.

Here, we try to solve this issue from the point of materials choosing and the design of device structure. Firstly, the applied polymers (blue, green and red) have the similar chemical structure and their EL spectra have an equidistant distribution. Obviously, similar chemical structure can ensure the excellent consistency to suppress the phase separation, which benefits to the spectra stability especially for the high driving current $[27,28]$. In addition, the latter will be conducive to obtain relatively flat EL spectra through the whole spectral range without pretty high crest and deep trough, which can guarantee the white emission with high CRI considering that the spectrum of sunlight is pretty flat in the visible spectrum. In addition, considering that the currentdependence spectrum mainly stems from the charge trapping, it should be a well strategy that designing a suitable device structure increase the direct charge injection to alleviate the effects of spectra stability from the charge trapping.

In this manuscript, three different type of devices were fabricated with the blend polymers (PF-blue, PF-green and PF-red) based benzothiophene-S, Sdioxide derivatives as the emissive layer. By introducing proper interface layer, the efficient, stable and high CRI WPLED was obtained due to the increasing emission from the direct charge injection and thus retarding the emission deriving from the charge trapping.

\section{Experimental section}

\subsection{Materials}

The PEDOT: PSS and TPBI were purchased from H. C. Starck and Luminescence Technology Corp, respectively. The polyvinyl carbazole (PVK), 2,2 ${ }^{0}$-(1,3-Phenylene)-bis [5-(4-tert- butylphenyl)-1,3,4- oxadiazole] (OXD-7) and cesium fluoride ( $\mathrm{CSF}$ ) were purchased from Sigma-Aldrich. The PF-blue, PF-green and PF-red which were synthesized according to the reported procedures [28,29], and the corresponding chemical structure were shown in Fig. 1a.

\subsection{Device fabrication}

The ITO glass substrates with a sheet resistance of $15-20 \Omega /$ square were

(a)

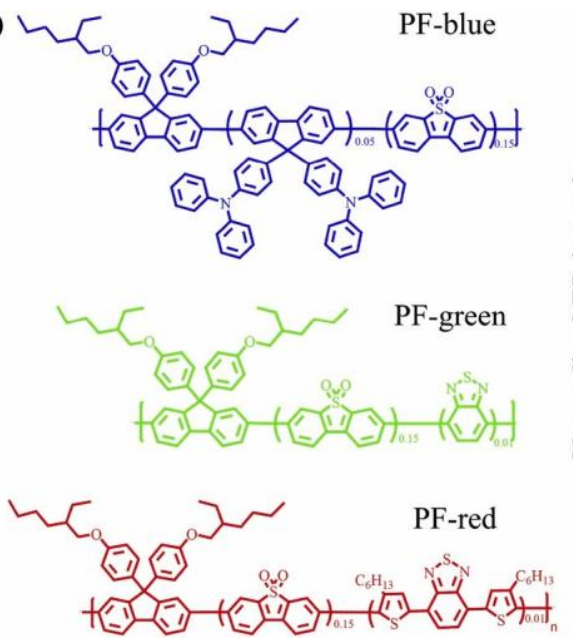

were spin-coated from its $\mathrm{p}$-xylene solution to form a uniform $80 \mathrm{~nm}$-thick film on top of the PEDOT: PSS layer, then annealed at $100 \mathrm{C}$ on a hotplate for $20 \mathrm{~min}$. Finally, a thin layer of CSF ( $1.5 \mathrm{~nm}$ thick) and $120 \mathrm{~nm}$ aluminum layer were evaporated with a shadow mask at a pressure of 3.0 $10^{4} \mathrm{~Pa}$. Except for the different annealing temperature $(80 \mathrm{C}, 100 \mathrm{C}, 120 \mathrm{C}, 140 \mathrm{C}$ and $160 \mathrm{C})$ of emissive layer, the devices with different annealing temperature were fabricated according to the same condition as described above. For the multilayer device, the hole transporting layer PVK $(40 \mathrm{~nm})$ was processed by spin- coating its chlorobenzene solution $\left(10 \mathrm{mg} \mathrm{ml}^{1}\right)$, then annealed at $120 \mathrm{C}$

on a hotplate for $10 \mathrm{~min}$. The electron transporting layer TPBI $(30 \mathrm{~nm})$ was evaporated at an evaporation rate of $0.1-0.8 \mathrm{~A} \mathrm{~s}^{1}$ under a pressure of 3.010 ${ }^{4} \mathrm{~Pa}$. The overlapping area between the cathode and anode defined the pixel size of $19 \mathrm{~mm}^{2}$. Except for the deposition of the PEDOT: PSS layer, all the processes were carried out in the controlled atmosphere of a nitrogen drybox (Vacuum Atmosphere Co.) containing less than 10 ppm oxygen and moisture.

\subsection{Measurements}

The thickness of the PEDOT: PSS and light-emitting layer were determined by A Tencor Alfa-step 500 Surface Profiler. The thickness of the TPBI and the evaporated cathodes were monitored by a quartz crystal thickness/ratio monitor (Model:STM-100/MF, Sycon). The current density-luminance-voltage $(\mathrm{J}-\mathrm{L}-\mathrm{V})$ characteristics were measured in the nitrogen dry-box using a Keithley 236 source-measurement unit and a calibrated silicon photodiode. The luminance was calibrated using a PR-705 SpectraScan spectrophotometer (Photo Research). The electroluminescent spectra (EL) and CIE coordinate was collected by the PR- 705 SpectraScan spectrophotometer while the CRI was calculated following the CIE publication released in 1974 [30]. Unless otherwise indicated, the EL spectra of all the devices were measured based on a constant current density $\left(10 \mathrm{~mA} \mathrm{~cm}{ }^{2}\right)$. The EQE values were calculated assuming a Lambertian emission profile. The UV-vis absorption spectra of the films were recorded using a SHIMADZU UV-2600 spectrophotometer. Photoluminescence $(\mathrm{PL})$ spectra were recorded using a HORIBA scientific fluoromax-4 spectrofluorometer.

\section{Results and discussions}

The chemical structures of PF-blue, PF-green and PF-red are shown in Fig. $1 \mathrm{a}$. It can be clearly seen that the three polymers possess similar chemical structure containing the 9,9-bis(4-(2-ethylhexyloxy) phenyl) fluorene unit and

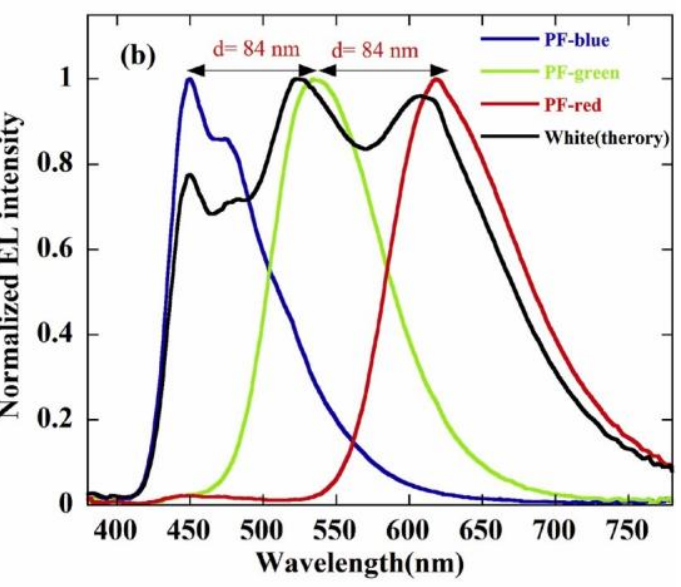

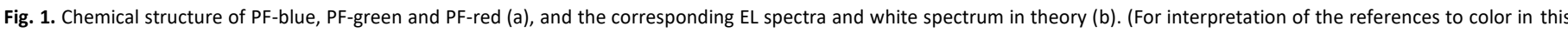
figure legend, the reader is referred to the Web version of this article.)

cleaned in an ultrasonic bath successively in acetone, detergent, deionized water, and isopropanol. After oxygen plasma treatment, a $40 \mathrm{~nm}$-thick PEDOT: PSS was spin-coated directly on the ITO substrate, then annealed at $120 \mathrm{C}$ on a hotplate for $20 \mathrm{~min}$ to remove the residual moisture. The polymer blend (PF-blue: PF-BT: PF-red) with different blends proportion
benzothiophene-S, S-dioxide unit which benefits to form homogeneous blend. Based on a simple conventional device structure of ITO/PEDOT: PSS (40 $\mathrm{nm}) / \mathrm{EML}(80 \mathrm{~nm}) / \mathrm{CsF}(1.5 \mathrm{~nm}) / \mathrm{Al}(120 \mathrm{~nm})$, the corresponding EL spectra were obtained, which could cover the entire visible light spectrum (Fig. 1b). The corresponding CIE coordinates of PF-blue, PF-PF-green and red are 
$(0.16,0.20),(0.35,0.58)$, and $(0.61,0.37)$, respectively. As depicted in Fig. $1 \mathrm{~b}$, the main peak of EL spectra $\left(\lambda_{E L}\right)$ located at $450 \mathrm{~nm}, 534 \mathrm{~nm}$ and $618 \mathrm{~nm}$, respectively. An interesting coincidence is that these EL spectra present an equidistant distribution. More specifically, the $\lambda_{\mathrm{EL}}$ of between the PF- blue and PF-green $84 \mathrm{~nm}$ interval, so does between the PF-green and PF- red. Furthermore, the corresponding half peak width are as high as $78 \mathrm{~nm}, 88 \mathrm{~nm}$ and $104 \mathrm{~nm}$, respectively. Such an equidistant distribution as well as the broader half peak width should be beneficial to acquire high CRI white emission. For instance, if we assume the blue, green and red have the same EL intensity, then it can obtain the white spectrum in theory by calculating the sum of EL intensity at one point (Fig. 1b). Furtherly, we could acquire its CIE $(0.36,0.38)$ and correlated color temperature (CCT: $4731 \mathrm{~K}$ ) by calculation through the Spectrawin software (Photo Research Inc.). In addition, the CRI of 93 also could be calculated following the publication method [30]. Therefore, the three polymers have a great probability to obtain white emission with high CRI (>90) through carefully controlling the doping ratio.

Hence, a series of type A devices with a configuration of ITO/PEDOT: PSS $(40 \mathrm{~nm}) / \mathrm{EML}(80 \mathrm{~nm}) / \mathrm{CsF}(1.5 \mathrm{~nm}) / \mathrm{Al}(120 \mathrm{~nm})$ were fabricated based on various doping ratios of the three polymers. Fig. 2 and Table 1 presents the relative device data of WPLEDs with doping ratios of 90:7:3 (W73), 87:7:6 (W76) and 88:6:6 (W66) for PF-blue, PF-green and PF- red, the corresponding device were named $A_{w 73}, A_{w 76}$ and $A_{w 66}$. Although all of them present a white emission, it's surprised that the doping ratio is pretty high compared with

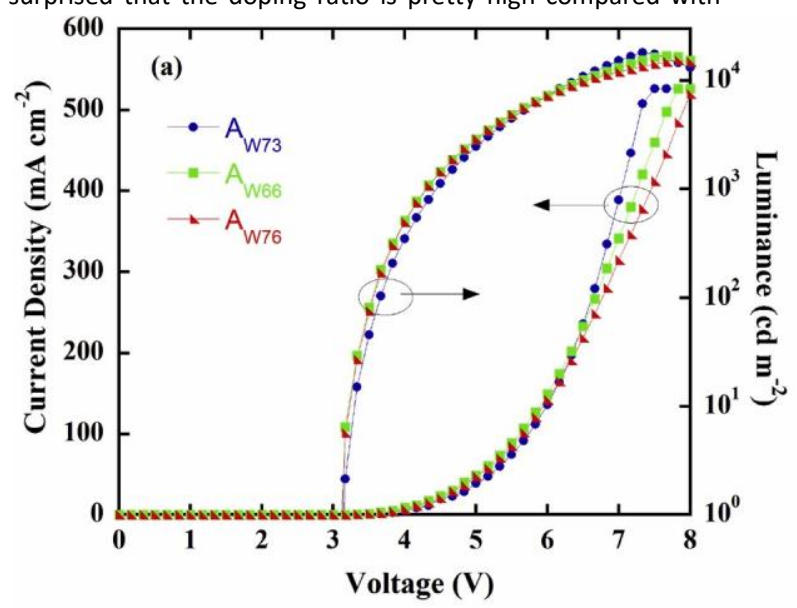

previous reports of doping ratio below $1 \mathrm{wt} \%[18,19]$. Even if the content of PF-red reach $3 \mathrm{wt} \%$, the emission from the PF-red is still very weak according to the corresponding EL spectrum (in Fig. 2c). It seems the energy transfer from the PF-blue to PF-red is blocked. Along with the content of PF-red reached 6

wt\%, the device ( $\left.A_{w 76}\right)$ realized a well-balanced white emission with high CRI of 88. The corresponding maximum LE (LE $\max$ ) was $8.04 \mathrm{~cd} \mathrm{~A}{ }^{1}$ and $\mathrm{CIE}$ coordinates of $(0.320,0.363)$ was obtained at a current density of $10.53 \mathrm{~mA}$ $\mathrm{cm}^{2}$. Besides, the thermostability of EL spectra were measured based on different annealing temperature ranging from $80 \mathrm{C}$ to $160 \mathrm{C}$, the EL spectra almost kept the same except the slight increase emission from the long wavelength which maybe stem from the enhanced charge transfer from the PF-blue to PF-green/PF-red due to the well-ordered packing of polymer chains (Fig. S1). Previous study also showed similar phenomenon [31]. Although the white emission possesses a better CRI (88), but it should have the potential for further improvement especially for the emission from the PF-red compared with the white spectrum in theory in Fig. 2c.

Therefore, it is very essential to uncover the white emission mechanism in this system for the further improvement. The most common fact is that there exist two independent mechanisms of energy transfer and charge trapping for the white emission. As shown in Fig. 3, the PL spectrum of PF-blue has hardly any overlap with the absorption

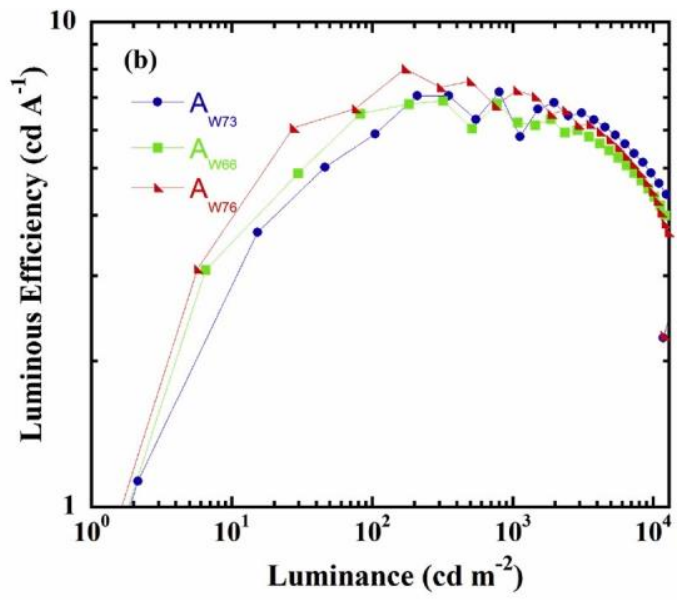

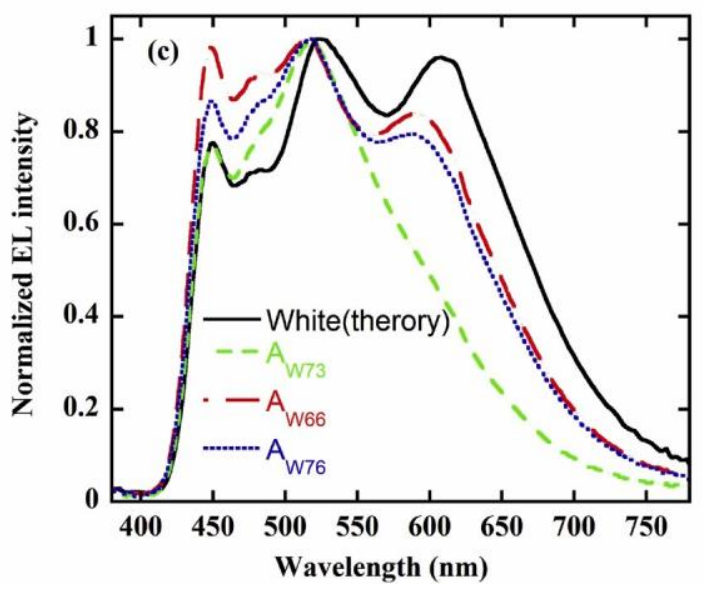

Table 1

Summary of key device performance metrics.

\begin{tabular}{|c|c|c|c|c|c|c|c|c|}
\hline Device & $\operatorname{LEmax}(\mathrm{cd} \mathrm{A} 1)$ & $P E_{\max }(\operatorname{Im~W} 1)$ & $\mathrm{EQE}_{\max }(\%)$ & $\operatorname{Lmax}(\mathrm{cd} \mathrm{m} \mathrm{2})$ & CCT (K) & CRI & ${ }^{\mathrm{a}} \mathrm{CIE}(\mathrm{x}, \mathrm{y})$ & $b V_{\text {th }}(\mathrm{V})$ \\
\hline Aw73 & 7.18 & 5.79 & 3.09 & 18156 & 7353 & 73 & $(0.282,0.383)$ & 3.17 \\
\hline Aw66 & 6.89 & 5.82 & 3.32 & 16903 & 5924 & 91 & $(0.322,0.355)$ & 3.17 \\
\hline Aw76 & 8.04 & 6.88 & 3.78 & 15377 & 6106 & 88 & $(0.320,0.363)$ & 3.17 \\
\hline Bw76 & 9.56 & 3.85 & 4.82 & 16876 & 5234 & 93 & $(0.339,0.353)$ & 6.60 \\
\hline
\end{tabular}




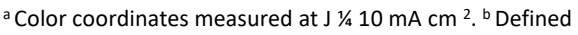
as the voltage at a luminance of $1 \mathrm{~cd} \mathrm{~m}^{2}$.

spectrum of either PF-green or PF-red, which means that energy transfer from PF-blue to PF-green (PF-red) should be invalid in this polymer blend system. Furthermore, the blend film still maintains stronger blue emission according to the PL spectra of the blend film despite the high doping ratio of PF-green and PF-red, it also can further verify the above inference (Fig. 3). Previous study also presented the similar phenomenon by using the similar polymer system [28,32]. The weak emission from PF-green and PF-red should mainly derive from the direct photoluminescence rather than the energy transfer from the PF-blue. Besides, that the current decreased along with the increased content of guest materials also provided the evidence about the existence of charge trapping (Fig. 2a) [23,33]. Therefore, it can be reasonable to speculate that the white emission should stem from the charge trapping, which require the highest occupied molecular orbital (HOMO) or the lowest unoccupied molecular orbital (LUMO) levels of guest materials (PF-green and PF-red) lying in the band gap of host material (PF-blue).

As depicted the energy level of WPLED in Fig. 4, the LUMO and HOMO of PF-blue, PF-green, PF-red are $2.55 \mathrm{eV} / 5.38 \mathrm{eV}, 3.05 \mathrm{eV} /$

$5.89 \mathrm{eV}$ and $3.90 \mathrm{eV} / 5.98 \mathrm{eV}$, respectively $[28,29]$. Obviously, there exists a $0.46 \mathrm{eV} / 1.34 \mathrm{eV}$ electron trap depth for the LUMO level between PF-blue and PF-green/PF-red. Therefore, for the electron injection of PF-green and PF-red, direct electron trapping in the green and red emitting polymers could occur. For the hole should be mainly injected from the HOMO level of PF-blue due to bigger injection barrier injection from the PEDOT: PSS. To further illustrate the situation of hole injection of PF-green and PF-red in this white light system, 30 wt\% electron transporting material (OXD-7) was added to the polymer blend system (W76), the corresponding EL was shown in Fig. S2. Obviously, its emission from the PF-green and PF-red were strongly restrained by adding the OXD-7 and showed the similar spectrum as the PL spectrum of W76. The emissions from PF-green and PF-red were suppressed because the OXD-7 could block the hole transporting from the HOMO

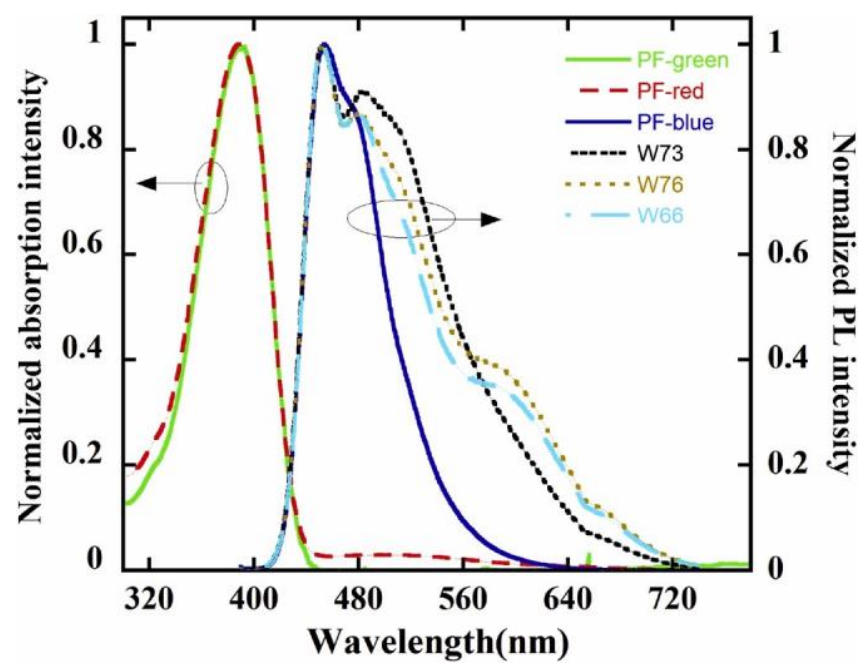

Fig. 3. Absorption spectra of PF-green and PF-red film, PL spectra of PF-blue, W73, W76 and $W 66$ film. (For interpretation of the references to color in this figure legend, the reader is referred to the Web version of this article.)

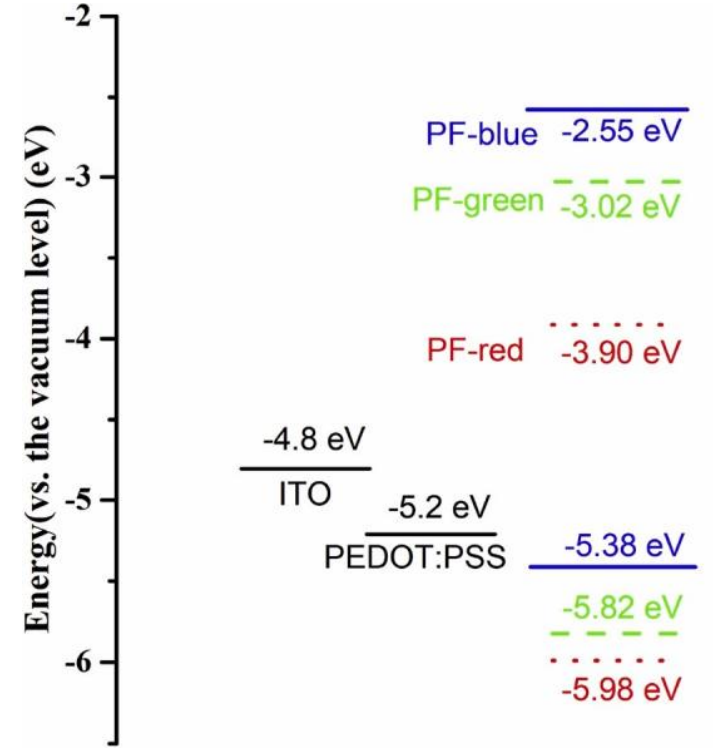

Fig. 4. Energy level diagram of WPLED.

level of PF-blue to PF-green and PF-red due to its deep HOMO level of $6.5 \mathrm{eV}$. The weak emission from PF-green and PF-red should stem from the direct electron and hole injection so that it presented the similar spectrum as the PL spectrum. Therefore, it could be reasonable that we can improve the emission from the PF-red by increasing the direct charge injection.

Considering the bigger hole injection barrier between the PEDOT: PSS ( 5.2 $\mathrm{eV}$ ) and PF-red (HOMO: $5.99 \mathrm{eV}$ ), the hole transporting layer PVK (HOMO: 5.6 $\mathrm{eV}$ ) was used to improve the direct hole injection for PF-red. Therefore, type $B$ device with the configuration of ITO/PEDOT: PSS (40 nm)/PVK $(40 \mathrm{~nm}) / E M L$ $(80 \mathrm{~nm}) / \mathrm{CsF}(1.5 \mathrm{~nm}) / \mathrm{Al}(120 \mathrm{~nm})$ was fabricated. As shown in Fig. S3a, the current density of Bw76 decreased with the introduce of PVK, which should owe to the lower hole mobility of PVK. For the device $B_{w 76}$, the $L_{\max }$ of $9.56 \mathrm{~cd} \mathrm{~A}^{1}$ shows a distinctly increased due to the enhancement of direct hole injection (Table 1 and Fig. S3). It's worth mentioning that the white light characteristic parameters of $\operatorname{CIE}(0.339,0.353)$ and CRI (93) got obvious improvement compared with that of device Aw76 due to the increased emission from PF-red. But the power efficiency decreased to $3.85 \mathrm{Im} \mathrm{W}{ }^{1}$ due to the increased $V_{\text {th }}$ 
The color-stability upon different driving current is also the key factor for general lighting. As shown in Fig. $5 a$ and $b$, both the type $A$ and type $B$ device present the similar variation tendency that the relative emission intensity from the trap site (PF-green and PF-red) gradually decrease along with the increased of driving current, such phenomenon can be observed widely in the charge trap system. The main reason is that the carriers injected from the electrodes were mainly distributed on the LUMO of guest due to the trap effect, as the drive current increases, the injected carrier's may tend to saturate on the guest sites [22]. Since the current dependence of spectra mainly depends on the electron injection from the charge trap in this white
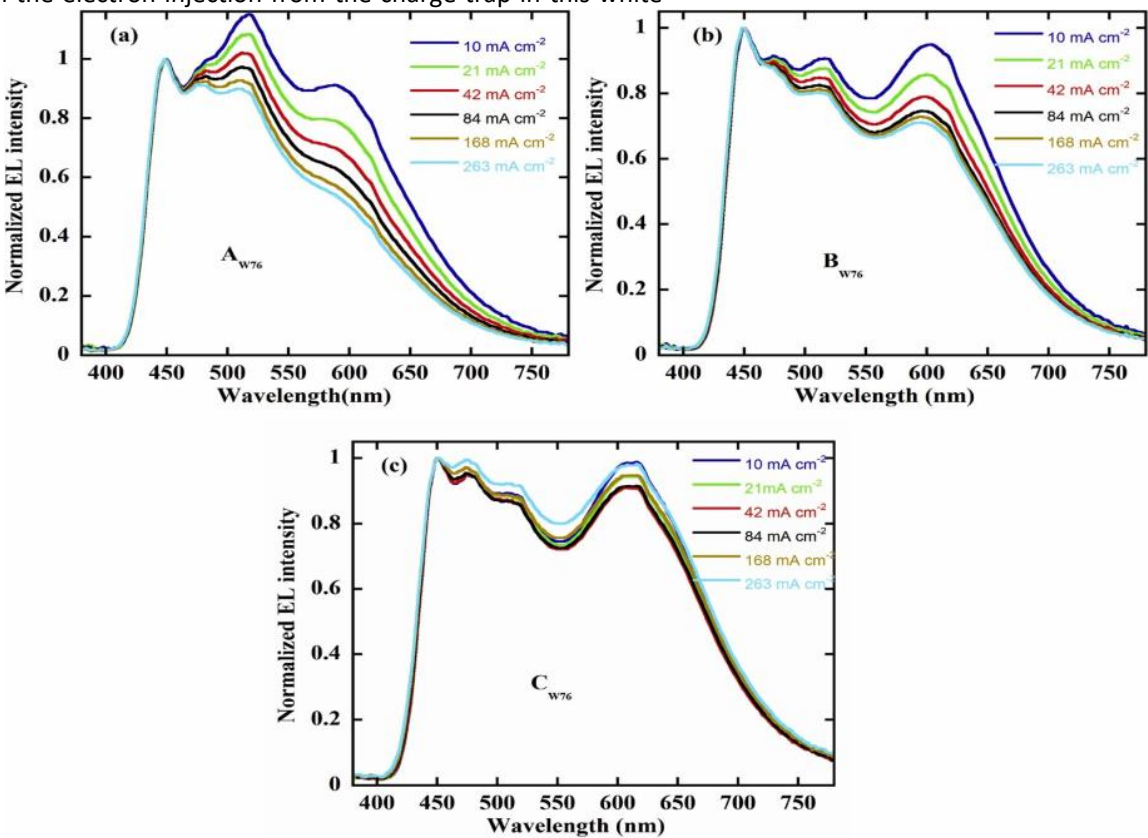

Fig. 5. EL spectra of $A_{W 76}$ device (a), $B_{W 76}$ device (b) and $C_{W 76}$ device (c) based on different driving current. light system, it means that the current dependence may be restrained if the direct electron injection can be enhanced for PF-red. To increase the direct electron injection should be feasible considering that the high content of PFred ( $6 \mathrm{wt} \%$ ) and the enhanced direct hole injection by PVK.

Previous study demonstrated that the electron transporting material TPBI can effectively improve the performance of PF-red due to the increased electron transporting and hole-blocking ability [28]. Therefore, type $C$ device with the configuration of ITO/PEDOT: PSS (40 nm)/EML (80 nm)/TPBI (30 $\mathrm{nm}) / \mathrm{CsF}(1.5 \mathrm{~nm}) / \mathrm{Al}(120 \mathrm{~nm})$ was fabricated. Compared with the device Aw76, the current density of device $\mathrm{C}_{w 76}$ also decreased which should be derived from the hole blocking of TPBI that could decrease the hole leak current (Fig. $\mathrm{S} 3 \mathrm{a})$. As expected, the $\mathrm{LE}_{\max }$ of the device $\mathrm{C}_{\text {w87 }}$ increased to $10.17 \mathrm{~cd} \mathrm{~A}^{1}$ because of the improvement of electron injection (Table 1 and Fig. S3). Moreover, it exhibits excellent stability of device performance, even if the luminance increased to $1000 \mathrm{~cd} \mathrm{~m}{ }^{2}$, the corresponding LE could still maintain at $9.68 \mathrm{~cd}$ $A^{1}$. Most satisfyingly, the color quality of the device was also greatly improved deriving from the enhanced emission from the PF-red (Fig. S4). The CIE coordinates of $(0.346,0.345)$ was reached, which is very close to equi-energy white point $(0.33,0.33)$. Due to the full width at half-maximum of the emission spectrum of the device was found to be about $248 \mathrm{~nm}$, which could effectively cover the full visible region and the $\mathrm{CRI}$ could reach as high as 92 . Furthermore shifted from $(0.346,0.345)$ to $(0.341,0.344)$ with CIE variations as small as $\Delta x$, $\Delta y 1 / 40.008,0.005$. The CRI values of the $C_{W 76}$ and $B_{w 76}$ device almost kept the same with the

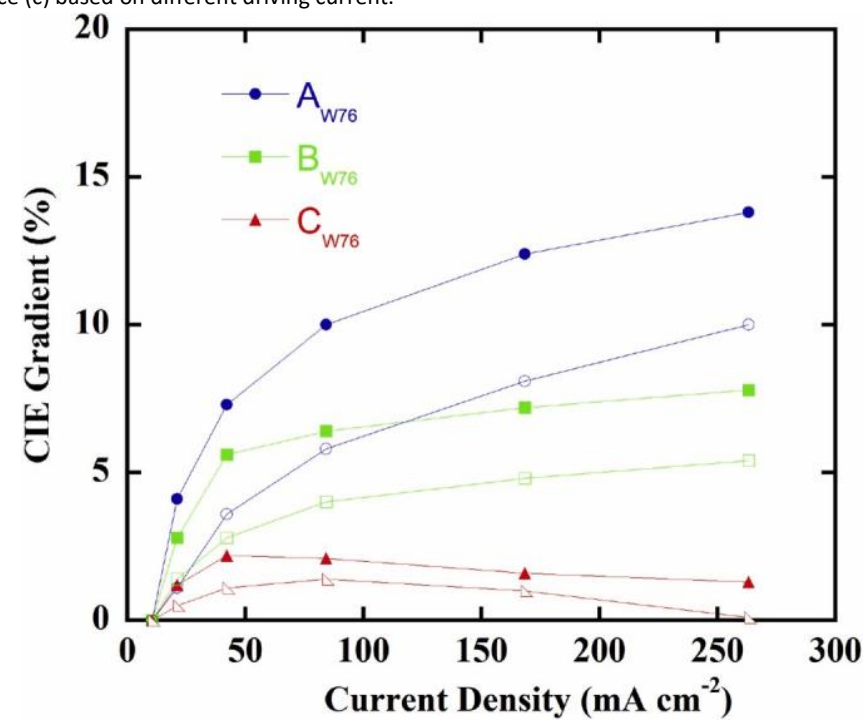

Fig. 6. CIE gradient vs current density curves for different device structure. Solid tag represents the gradient of $\mathrm{CIE}(\mathrm{x})$, and the empty tag represent the gradient of $\mathrm{CIE}(\mathrm{y})$.
Fig. 7. AFM height images (5 $5 \mu \mathrm{m}$ ): the PF-blue film (a) and the blend film (W76) (b). (For interpretation of the references to color in this figure legend, the reader the color temperature (CCT) of $4942 \mathrm{~K}$ is very close to the CCT of standard daylight at solar altitude $20(4700 \mathrm{~K})$ [34]. Therefore, this device could be fit for solid-state lighting sources.

At last, we turn our attention to the color-stability for the device $\mathrm{C}_{\text {w76. }}$. It can be seen that this device shows excellence color-stability and the spectra almost keep the same with the driving bias increased from $10 \mathrm{~mA} \mathrm{~cm}{ }^{2}$ to 263 $\mathrm{mA} \mathrm{cm}{ }^{2}$ (Fig. 5c). As shown in Table S1, the corresponding $\mathrm{CIE}$ coordinates is referred to the Web version of this article.)

current intensity increased from $10 \mathrm{~mA} \mathrm{~cm}{ }^{2}$ to $263 \mathrm{~mA} \mathrm{~cm}{ }^{2}$, while that of the Aw76 device decreased from 88 to 85 .

Specially, to visually and concretely present the variation of spectra, the gradient of CIE coordinates vs current density curve for the devices were shown in Fig. 6. The CIE variation of $A_{W 76}$ and $B_{W 76}$ device were beyond $5 \%$ even 
as high as $14 \%$, meanwhile, it could be controlled around $2 \%$ for the $\mathrm{C}_{\text {w76 }}$ device (Fig. 6 and Table S1). The main reason should be that the electron injection origin from the electron trapping was restrained due to the enhanced electron injection from the TPBI filling the LUMO site. Since the current dependence of spectra mainly depends on the electron injection from the charge trap, it means that dependence may be restrained if the direct electron injection rather than from the trapping can be enhanced for PF-red.

The high CRI and spectrum stability of device $\mathrm{C}_{\text {w76 }}$ was attributed to not only the appropriate device structure but also the good compatibility of the polymer blend. These polymers have similar chemical structure which can effectively suppress phase separation at high current density. The similar rootmean-square surface roughness of the PF-blue film $(0.592 \mathrm{~nm})$ and the blend film (W76) ( $0.581 \mathrm{~nm}$ ) obtained by the atomic force microscopy (AFM) height images closely matches the expectation (Fig. 7).

\section{Conclusions}

To summarize, we obtained stable, efficient WPLEDs as well as excellent $\mathrm{CRI}$ based on polymer blend by restraining the electron trapping. The polymers (blue, green and red) in blend film having a similar chemical structure and equidistant distribution EL spectra were benefited to acquire high CRI and stable spectra for WPLEDs. By optimizing the device configuration to enhance the direct injection, the high efficient (10.17 cd A ${ }^{1}$ ), high CRI (92) and high color stability $(0.3420 .004,0.3430 .002)$ WPLED was obtained.

\section{Declaration of competing interest}

The authors declare that they have no known competing financial interests or personal relationships that could have appeared to influence the work reported in this paper.

\section{Acknowledgements}

This work was supported by Guangdong Basic and Applied Basic Research Foundation, China (No. 2019A1515110602), Scientific Research Foundation of Advanced Talents (innovation team) of DGUT, China (No. KCYCXPT2016004), Scientific Research Foundation for Young Team of DGUT, China (No. TDQN2019005), Department of Science and Technology of Guangdong Province, China (No. 2020A151501814), and Department of Education of Guangdong Province, China (No. 2018KTSCX223).

\section{Appendix A. Supplementary data}

Supplementary data to this article can be found online at https://doi. org/10.1016/j.orgel.2020.105785.

\section{References}

1. Zhang, Y. F.; Xu, Z.; Zhang, F. J.; Wang, Y.; Zhao, S. L., White organic light emitting device with dyestuff DCJTB mended in polymer. Spectroscopy and Spectral Analysis 2008, 28 (4), 760-762.

2. Watanabe, K.; Sakamoto, T.; Taguchi, M.; Fujiki, M.; Nakano, T., A chiral pi-stacked vinyl polymer emitting white circularly polarized light. Chem. Commun. 2011, 47 (39), 10996-10998.

3. Wang, R.; Peng, J.; Qiu, F.; Yang, Y. L., Enhanced white-light emission from multiple fluorophores encapsulated in a single layer of diblock copolymer micelles. Chem. Commun. 2011, 47, 2787-2789

4. Prakash, A.; Katiyar, M., White polymer light emitting diode using blend of fluorescent polymers. 16th International Workshop on Physics of Semiconductor Devices 2012, 8549.

5. Park, J. J.; Park, T. J.; Jeon, W. S.; Kim, S. Y.; Lee, Y. K.; Jang, J.; Kwon, J. H., White Polymeric Light-Emitting Diodes Based on Doping of an Orange Ir Complex in a Fluorene Blue Polymer Host. Mol. Cryst. Liq. Cryst. 2009, 498, 290-297.
6. Park, J. J.; Park, T. J.; Jeon, W. S.; Kim, S. Y.; Lee, Y. K.; Jang, J.; Kwon, J. $\mathrm{H}$., White polymeric light-emitting diodes based on doping of an orange Ir complex in a fluorene blue polymer. Idw '07: Proceedings of the 14th International Display Workshops, Vols 1-3 2007, 1033-1036.

7. Nicolai, H. T.; Hof, A. J.; Blom, P. W. M., Charge transport in white lightemitting polymers. Organic Optoelectronics and Photonics li 2006, 6192.

8. Nicolai, H. T.; Hof, A.; Blom, P. W. M., Device Physics of White Polymer Light-Emitting Diodes. Adv. Funct. Mater. 2012, 22 (10), 2040-2047.

9. Li, B. N.; Liu, L.; Fu, G. R.; Zhang, Z.; Li, H. Y.; Lu, X.; Wong, W. K.; Jones R. A., Color-tunable to direct white-light and application for white polymer light emitting diode (WPLED) of organo-Eu3+- and organo-Tb3+-doping polymer. J. Lumin. 2017, 192, 1089-1095.

10. Shen, F. Z.; He, F.; Lu, D.; Xie, Z. Q.; Xie, W. J.; Ma, Y. G.; Hu, B., Bright and colour stable white polymer light-emitting diodes. Semiconductor Science and Technology 2006, 21 (2), L16-L19.

11. Kim, C.; Gwon, Y. J.; Kim, J.; Lee, T. S., Synthesis of fluorescent conjugated polymer nanoparticles and their immobilization on a substrate for white light emission. Polym Chem-Uk 2018, 9 (48), 5671-5679.

12. Amin, G.; Zaman, S.; Zainelabdin, A.; Nur, O.; Willander, M., ZnO nanorods-polymer hybrid white light emitting diode grown on a disposable paper substrate. Physica Status Solidi-Rapid Research Letters 2011, 5 (2), 71 73.

13. Taudt, C.; Baselt, T.; Oreski, G.; Hirschl, C.; Koch, E.; Hartmann, P., Cross-linking characterization of polymers based on their optical dispersion utilizing a white-light interferometer. Optical Measurement Systems for Industrial Inspection Ix 2015, 9525.

14. Song, H. J.; Shin, G. J.; Choi, K. H.; Lee, S.; Moon, D. K., White polymer light emitting diode materials introducing dendritic quinoxaline derivative: Synthesis, optical and electroluminescent properties. Synthetic Metals 2014, 190, 1-7.

15. Lee, H. K.; Kim, T. H.; Park, J. H.; Kim, J. K.; Park, O. O., White-lightemitting diodes using miscible polymer blend doped with phosphorescent dye. Organic Electronics 2011, 12 (6), 891-896.

16. Kim, J. H.; Song, W. S.; Yang, H., Color-converting bilayered composite plate of quantum-dot-polymer for high-color rendering white light-emitting diode. Opt. Lett. 2013, 38 (15), 2885-2888.

17. Kassamakov, I.; Ojala, K.; Salmia, A.; Haeggstrom, E.; Aaltonen, J.; Huber, A.; Saarikko, H.; Osterberg, M.; Oinonen, M., Characterization of dents and grooves on polymer films using scanning white light interferometry. Optical Micro- and Nanometrology in Microsystems Technology 2006, 6188.

18. Inoue, A.; Hosokawa, T.; Haishi, M.; Ohtani, N., 4-(dicyanomethylene)2-methyl-6-(p-dimethylaminostyryl)-4H-pyran (DCM)-doping density dependence of luminescence spectra and white emission in polymer lightemitting diodes. Physica Status Solidi C - Current Topics in Solid State Physics, Vol 6, No 1 2009, 6 (1), 334-337.

19. Hu, B.; Yao, C.; Huang, X. R., Designing of the White-Light Emission from a Single-Polymer System: Quantum Theoretical Study. Polymer Science Series A 2011, 53 (11), 1097-1105.

20. Fan, L. M.; Fan, W. L.; Li, B.; Zhao, X.; Zhang, X. T., W-shaped 1,3-di(2,4dicarboxyphenyl)benzene based lanthanide coordination polymers with tunable white light emission. New J. Chem. 2016, 40 (12), 10440-10446. 21. da Silva, M. A. T.; Thomazini, E. F.; Albertini, M.; Renzi, W.; Franchello, F.; Dias, I. F. L.; Duarte, J. L.; Pocas, L. C.; Lourenco, S. A., Characterization of digital textile printing and polymer blend (PFO-DMP:P3HT) for application in manufacture of organic diodes emitting white light - WOLEDS. Optical Materials 2016, 62, 119-131.

22. Chen, M. X.; Sun, R. Y.; Ye, Y. C.; Tang, H. J.; Dong, X. Y.; Yan, J. L.; Wang, K. M.; Zhou, Q.; Wang, Z. L., Application of a novel red-emitting cationic iridium(III) coordination polymer in warm white light-emitting diodes. Optical Materials 2018, 76, 141-146.

23. Wang, R.; Peng, J.; Qiu, F.; Yang, Y. L.; Xie, Z. Y., Simultaneous blue, green, and red emission from diblock copolymer micellar films: a new approach to white-light emission. Chem. Commun. 2009, (44), 6723-6725. 24. Hrma, M.; Sichova, K.; Svoboda, J.; Vohlidal, J., Assembling of bis(tpy)fluorenes with $\mathrm{Zn} 2+$ and Fe2+ ions into metallo-supramolecular polymers with highly efficient white-light emission. Polymer 2017, 122, 2233.

25. Chitara, B.; Bhat, S. V.; Vivekchand, S. R. C.; Gomathi, A.; Rao, C. N. R., White-light sources based on composites of GaN nanocrystals with 
conducting polymers and nanophosphors. Solid State Communications 2008, 147 (9-10), 409-413.

26. Cheng, G.; Fei, T.; Zhao, Y.; Ma, Y. G.; Liu, S. Y., White phosphorescent polymer light-emitting devices based on a wide band-gap polymer derived from 3,6-carbazole and tetraphenylsilane. Organic Electronics 2010, 11 (3), 498-502.

27. Chen, S. A.; Chang, E. C.; Chuang, K. R.; Chao, C. I.; Wei, P. K.; Fann, W. S., Conjugated polymer blends as emitting layer for white light led. Abstr. Pap. Am. Chem. Soc. 1998, 215, U392-U392.

28. Zhen, H. Y.; Xu, W.; King, W.; Chen, Q. L.; Xu, Y. H.; Jiang, J. X.; Peng, J. B.; Cao, Y., White-light emission from a single polymer with singlet and triplet chromophores on the backbone. Macromol. Rapid Commun. 2006, 27 (24), 2095-2100.

29. Zhang, T. H.; Gong, Z. C.; Que, L., A white-light source operated polymer-based micromachined Fabry-Perot chemo/biosensor. 2009 4th leee International Conference on Nano/Micro Engineered and Molecular Systems, Vols 1 and 2 2009, 181-184.

30. Willander, M.; Nur, O.; Zaman, S.; Zainelabdin, A.; Bano, N.; Hussain, I., Zinc oxide nanorods/polymer hybrid heterojunctions for white light emitting diodes. J. Phys. D: Appl. Phys. 2011, 44 (22).

31. Wang, Z. W.; Gao, D. Z.; Ma, X. J.; Meng, J., White-Light Interferometry for Measuring Fuel Pressure in Icf Polymer-Microsphere Targets. Fusion Sci. Technol. 2014, 66 (3), 432-437.

32. Wang, B. Z.; Zhang, X. P.; Liu, H. M., White-light-emitting diode based on a single-layer polymer. Aip Advances 2013, 3 (5).

33. Tian, L. L.; Zhang, W.; Yang, B.; Lu, P.; Zhang, M.; Lu, D.; Ma, Y. G.; Shen, J. C., Zinc(II)-induced color-tunable fluorescence emission in the piconjugated polymers composed of the bipyridine unit: A way to get whitelight emission. J. Phys. Chem. B 2005, 109 (15), 6944-6947.

34. Tang, K. C.; Tseng, S. R.; Li, W. S.; Meng, H. F.; Horng, S. F.; Hsu, C. S., Broad band and white phosphorescent polymer light-emitting diodes in multilayer structure. Synthetic Metals 2008, 158 (7), 287-291.

35. Sun, C.; Zhang, Y.; Sun, K.; Reckmeier, C.; Zhang, T. Q.; Zhang, X. Y.; Zhao, J.; Wu, C. F.; Yu, W. W.; Rogach, A. L., Combination of carbon dot and polymer dot phosphors for white light-emitting diodes. Nanoscale 2015, 7 (28), 12045-12050.

36. Roberts, R. J.; Le, D.; Leznoff, D. B., Color-Tunable and White-Light Luminescence in Lanthanide Dicyanoaurate Coordination Polymers. Inorg. Chem. 2017, 56 (14), 7948-7959.

37. Qin, L. J.; Zhu, Y. C.; Yang, H.; Ding, L.; Sun, F.; Shi, M.; Yang, S. P., White-light phosphorescence from binary coordination polymer nanoparticles. Mater. Chem. Phys. 2013, 139 (2-3), 345-349.

38. Ovens, J. S.; Christensen, P. R.; Leznoff, D. B., Designing Tunable WhiteLight Emission from an Aurophilic Cu-l/Au-I Coordination Polymer with Thioether Ligands. Chemistry-a European Journal 2016, 22 (24), 8234-8239. 39. Niu, Y. H.; Liu, M. S.; Ka, J. W.; Bardeker, J.; Zin, M. T.; Schofield, R.; Chi, Y.; Jen, A. K. Y., Crosslinkable hole-transport layer on conducting polymer for high-efficiency white polymer light-emitting diodes. Adv. Mater. 2007, 19 (2), 300-+.

40. Niu, W. Y.; Sun, J. W.; Yan, P. F.; Li, Y. X.; An, G. H.; Li, G. M., 2D I-Ditoluoyl-tartaric acid Lanthanide Coordination Polymers: Toward Singlecomponent White-Light and NIR Luminescent Materials. Chemistry-an Asian Journal 2016, 11 (4), 555-560.

41. Nam, G. H.; Park, I. K., CdSe Quantum dot-conducting polymer hybrid structure for Phosphor-free white light-emitting diodes. Journal of the Korean Physical Society 2015, 66 (5), 785-789.

42. Meng, L. C.; Lou, Z. D.; Yang, S. Y.; Hou, Y. B.; Teng, F.; Liu, X. J.; Li, Y. $B .$, White organic light-emitting diodes based on a combined electromer and monomer emission in doubly-doped polymers. Chinese Physics B 2012, 21 (8). 